Ana Sofía Saltos-Cedeño; Patricio Alfredo Vallejo-Valdivieso; María Elena Moya-Martínez http://dx.doi.org/10.35381/e.k.v3i5.723

\title{
Innovación en educación matemática de básica superior durante el confinamiento por COVID-19
}

\author{
Innovation in higher basic mathematics education during confinement \\ by COVID-19 \\ Ana Sofía Saltos-Cedeño \\ asaltos2041@pucem.edu.ec \\ Pontificia Universidad Católica del Ecuador, Extensión Manabí, Portoviejo \\ Ecuador \\ https://orcid.org/0000-0001-7244-3239 \\ Patricio Alfredo Vallejo-Valdivieso \\ pvallejo@pucem.edu.ec \\ patricio2871@yahoo.es \\ Pontificia Universidad Católica del Ecuador, Extensión Manabí, Portoviejo \\ Ecuador \\ https://orcid.org/0000-0003-3248-7864 \\ María Elena Moya-Martínez \\ mmoya@pucem.edu.ec \\ Pontificia Universidad Católica del Ecuador, Extensión Manabí, Portoviejo \\ Ecuador \\ https://orcid.org/0000-0001-7504-6933
}

Recepción: 20 abril 2020

Revisado: 20 de mayo 2020

Aprobación: 06 junio 2020

Publicación: 11 de junio 2020

\section{RESUMEN}

La investigación tiene por objetivo analizar la innovación en educación matemática de básica superior durante confinamiento por COVID-19 en el Ecuador. Metodológicamente fue de carácter exploratorio descriptivo con enfoque cuantitativo no experimental, los docentes que cumplieron los criterios de inclusión poblacional fueron 8. Los resultados se basaron en explorar las áreas relacionadas a: Matemática inclusiva. Actualización docente. Formación del estudiante. Innovación educativa. Entre las conclusiones se tiene que la innovación en educación matemática de básica superior durante confinamiento por COVID-19 en el Ecuador, debe centrarse en un 
aprendizaje mediado por TIC mediante metodologías centradas en el estudiante como aprendizaje basado en problemas, con lo cual, el docente tiene la posibilidad de diseñar estrategias en conformidad a las necesidades del entorno social donde habita el estudiante, para lo cual, puede desarrollar proyectos en donde se involucren las operaciones matemáticas en consonancia con los recursos disponibles para tal fin.

Descriptores: Tecnología de la información; aprendizaje activo; enseñanza asistida por ordenador; epidemiología. (Palabras tomadas del Tesauro UNESCO).

\begin{abstract}
The objective of the research is to analyze innovation in higher basic mathematics education during confinement by COVID-19 in Ecuador. Methodologically, it was descriptive exploratory in nature with a non-experimental quantitative approach. Eight (8) were the teachers who met the population inclusion criteria. The results were based on exploring the areas related to: inclusive mathematics, teaching update, student training and educational innovation. As a conclusion, the innovation in higher basic mathematics education during confinement by COVID-19 in Ecuador, should focus on ICT-mediated learning through student-centered methodologies such as problem-based learning, with which the teachers have the possibility of designing strategies in accordance with the needs of the social environment where the student lives, as well as, developing projects that involve mathematical operations by using the resources available for this purpose.
\end{abstract}

Descriptors: Information technology; activity learning; computer assisted instruction; epidemiology. (Words taken from the UNESCO Thesaurus).

\title{
INTRODUCCIÓN
}

La sociedad del tercer milenio en la cual vivimos, se caracteriza por cambios acelerados en el campo de la ciencia y tecnología: los conocimientos, las herramientas, las maneras de hacer y comunicar la matemática tienden a evolucionar; por esta razón, tanto el aprendizaje como la enseñanza de la Matemática deben estar enfocados en el desarrollo de las destrezas necesarias para que el estudiantado sea capaz de resolver problemas cotidianos, a la vez que se fortalece el pensamiento lógico y creativo (Travieso-Valdés \& Ortíz-Cardenas, 2018).

Aunado a lo planteado, las matemáticas representan históricamente un conocimiento 
Ana Sofía Saltos-Cedeño; Patricio Alfredo Vallejo-Valdivieso; María Elena Moya-Martínez

esencial para la vida humana, desde antiguo ha estado presente en la construcción de las diversas civilizaciones, piramides, ciudades, han sido algunas de las estructuras generadas a partir del cálculo matemático, sin embargo, culturalmente se ha posicionado como un aprendizaje dificil, no atractivo para gran parte de los estudiantes (Orrantia, 2005). Esto ha posibilitado la apertura de la enseñanza de las matemáticas en relación a la innovación educativa, incorporandose progresivamente estrategias didácticas que motivan al estudiante hacia el aprendizaje matematico, en este sentido (Ponce-Díaz, 2017), destaca que:

Si cambiamos la manera o el método de enseñar matemáticas, una mayor cantidad de estudiantes logrará adquirir el aprendizaje con facilidad. De hecho, el aprendizaje y la aplicación de las matemáticas desarrollan habilidades claves como la resolución de problemas, que sirven también para la comprensión de otras áreas de conocimiento como las ciencias ( $\mathrm{P}$. 1).

La educación hoy día, no ve las matemáticas como una disciplina aislada, sino, interconectada que posibilita la generación de aprendizajes en otras materias, proyectandose la generación de acciones pedagógicas en favor de promover un aprendizaje complejo e integrador de competencias (Calvo-Cereijo, 2019). Se promueve así, una educación en donde el estudiante tiene la posibilidad de innovar desde los recursos didácticos con los cuales cuenta.

Hoy día parte de estos recursos son conformados desde las TIC (Grisales-Aguirre, 2018), lo cual implica transcender la enseñanza tradicional hacia el apoyo en estrategias didácticas basadas en la tecnología. Así el estudiante asume un rol principal en el aprendizaje, desarrollando un rol investigador que le permite asumir una serie de competencias favorecedores para su crecimiento integral, como trabajo en equipo, autonomía, motivación al logro, (Ausín, et al, 2016). En este sentido, el sistema educativo del Ecuador, ha venido implementando transformaciones en su curriculo, siendo uno de ellos, la incorporación de las TIC en el aprendizaje de las matemáticas, especificamente en la etapa "educación básica superior" tal como se indica: 
Ol.4.8. Recopilar, organizar e interpretar materiales propios y ajenos en la creación científica, artística y cultural, trabajando en equipo para la resolución de problemas, mediante el uso del razonamiento lógico, fuentes diversas, TIC, en contextos múltiples y considerando el impacto de la actividad humana en el entorno (Ministerio de Educación del Ecuador, 2016).

Se proyecta la innovación como parte del proceso enseñanza - aprendizaje con enfasis al uso de TIC, siendo esto directo con la actual situación vivida a causa del confinamiento por COVID-19, donde el Estado Ecuatoriano, ha asumido el teletrabajo como opción para cumplir con las obligaciones de la administración pública, no estando exento la educación en todos sus niveles, constituyendose la modalidad presencial en virtual como elemento que contribuya a la prevención de la enfermedad.

El forzoso proceso de asumir la educación vritual por COVID-19, permite al docente enfrentarse al reto de planificar sobre la base de promover el aprendizaje en matemáticas desde la virtualidad, esto implica adecuar lo propuesto en el curriculo, situación para la cual, se ha previsto el plan educativo por COVID -19 (Creamer, 2020). Desde lo planteado, se debe propiciar estrategias innovadoras con la finalidad de promover el conocimiento significativo en los estudiantes, especialmente en matemáticas.

Esto permite en el docente generar capacidades para la creación e innovación, con fines de adecuarse al nuevo contexto virtual donde debe laborar, siendo necesario que conozca sobre el abordaje de estrategias, recursos didácticos, que contribuyan de modo efectivo el aprendizaje en mediación de las TIC (Jiménez-Galán, 2017), lo contrario sería improvisar sin fundamento pedagogico, originandose un desequilibrio que no favorece al óptimo aprendizaje.

Asi mismo, es necesario tener en cuenta la posibilidad de que existan estudiantes con afecciones neurobiológicas que puedan incidir negativamente en el aprendizaje de las matemáticas, opción a tener en consideración por parte del docente a la hora de diseñar las estrategias a implementar (De-La-Peña-Álvarez \& Bernabéu-Brotóns, 2018). Atender esta situación, posibilita minimizar el riesgo de deserción escolar a causa de la 
no comprensión de las matemáticas (Muñoz-Camacho, et al, 2018), tener en consideración factores que pueden influir en deserción escolar, permite proyectar la adecuación pedagógica para contar con un escenario educativo en donde exista menor posibilidad de fracaso, siendo esta una tarea a tener en cuenta por parte del docente, recurriendo a la innovación como parte estrategica para conformar una asertiva afrontación del aprendizaje (Pascua-Cantarero, 2016).

El diseño de los ambientes virtuales de aprendizaje como recurso didáctico, deben ser pensados para la multiplicidad de condiciones e intereses de los estudiantes, no puede ser líneal y uniforme por cuanto se corre el riesgo de excluir (Vega-Vega, Niño-Duarte \& Cárdenas, 2015), en este sentido, no puede perderse la concepción de integración de los estudiantes con sus pares, por cuanto posibilita el trabajo cooperativo, comunicación interpersonal, generandose la socialización desde la educación (Rodríguez Pautt, 2014).

La postura de (Islas-Torres, 2018), indica que "la sociedad del conocimiento exige cada vez más individuos con capacidades complejas mejor y altamente desarrolladas. Sin embargo, los estudiantes denominados millenials que se supone corresponderían a estas exigencias, aún distan de lo que en el discurso se plantea" (p. 13), siendo este factor a tener en cuenta por parte del docente, quien debe enfrentarse al reto de promover un aprendizaje matemático, en estudiantes que a pesar de ser nativos de la era digital, no han adquirido en muchos casos esta competencia, sumado al propio esfuerzo del docente de asumir la modalidad virtual, cuando inicialmente ha venido trabajando en lo presencial (Herrera, et al, 2018).

Creandose una oportunidad para promocionar el aprendizaje basado en TIC, tanto en docentes como estudiantes, como una alternativa para crecer en función de las tendencias globales que promueven una educación inclusiva desde lo tecnológico, aunado a tener encuenta, los recursos como internet, equipos, por parte del estudiante para asumir una determinada innovación que sea diseñada por parte del docente (SaidHung, Valencia-Cobos \& González-Prieto, 2017). 
Ana Sofía Saltos-Cedeño; Patricio Alfredo Vallejo-Valdivieso; María Elena Moya-Martínez

Así mismo, (Mendoza, Burbano \& Valdivieso, 2019) destaca el rol del docente en el aprendizaje de las matematicas, donde sus competencias deben estar asociadas al entorno virual, paratiendo de su experiencia, conocimiento especifico, con la finalidad de generar una integración que permita la configuración de una educación de calidad en conformidad a los retos planteados, de ese modo, se estará frente a la posibilidad de conciliar un conocimiento en conformidad a las politivas educativas del Ecuador, donde se requiere un aprendizaje inclusivo, innovador, en relación a los desafíos del país para su transformación socio económica en los próximos años (Calvo, 2015).

En relación a lo planteado, el (Instituto Nacional de Evaluación Educativa, 2018), destaca la inversión que ha venido realizando el Estado del Ecuador para contrarrestar la deserción escolar y elevar la calidad de la educación, es así que la situación actual por pandemia COVID-19, ha obligado a asumir lo virtual como alternativa a la presencialidad, no debe realizarse en detrimento de los indicadores de calidad, sino, es necesario elevarlos para constituir la situación adversa como un aprendizaje para el crecimiento de la sociedad ecuatoriana (Estrada, Monferrer \& Moliner, 2016).

Por lo tanto, las TIC como innovación educativa incide en la generación de un aprendizaje significativo en las matemáticas (Carranza-Alcántar, 2017), siendo necesario contar para tal fin, lo focalizado por los lineamientos del Ministerio de Educación del Ecuador, sumado al diseño por parte del docente de estrategias en conformidad a focalizar un aprendizaje inclusivo en pertinencia a los retos sociales globales (García-Navarro, Massani-Enríquez \& Bermúdez-López, 2016), en razón de lo descrito, se tiene por objetivo: analizar la innovación en educación matemática de básica superior durante confinamiento por COVID-19 en el Ecuador.

\section{MÉTODO}

La investigación fue de carácter exploratorio descriptivo con enfoque cuantitativo no experimental (Hernández, Fernández \& Baptista, 2014), siendo un trabajo de corte observacional, se procedió a implementar el esquema de guías STROBE, con fines de 
Ana Sofía Saltos-Cedeño; Patricio Alfredo Vallejo-Valdivieso; María Elena Moya-Martínez

brindar profundidad metódica al estudio realizado (Cartes-Velasquez \& Moraga, 2016), por consiguiente, se procedió del siguiente modo:

\section{Población y muestra de estudio}

La población estuvo conformada por 45 docentes de la Unidad Educativa Fiscomisional San Francisco de Sales, ubicada en la provincia de Manabí-Ecuador, en consideración a la muestra, estuvo conformada por quienes cumplieron los criterios de inclusión.

\section{Criterios de inclusión de la muestra}

Para ser parte de la muestra poblacional, los docentes debían cumplir con los siguientes requerimientos:

1. Ser docente activo de la Unidad Educativa Fiscomisional San Francisco de Sales.

2. Ser docente de matemáticas de básica superior.

3. Estar dispuesto a participar en la investigación, mediante la elaboración de la encuesta vía online.

Los docentes que cumplieron los criterios de inclusión poblacional fueron 8, constituyéndose así, en la muestra de investigación.

\section{Técnica e instrumento de recolección de información}

Se aplicó la técnica de la encuesta vía online (formulario Google), de ese modo, la muestra poblacional aportó información pertinente al tema de investigación, en conformidad de responder un instrumento tipo cuestionario de 13 ítems con cuatro alternativas de respuestas, las cuales se identificaron con valores del 1 al 4 en orden creciente como rango de importancia, lo cual permitió cumplir con las normativas preventivas por COVID-19, sin entorpecerse la recolección de información.

Así mismo se procedió a realizar una prueba piloto del instrumento con la finalidad de establecer la confiabilidad del instrumento para su respectiva aplicación, calculándose 
Ana Sofía Saltos-Cedeño; Patricio Alfredo Vallejo-Valdivieso; María Elena Moya-Martínez

el Alfa de Cronbach como coeficiente recurrente al tipo de cuestionario aplicado, procediéndose mediante la fórmula propuesta por (Hernández, Fernández \& Baptista, 2014), una vez obtenidos los datos de la muestra piloto, se procedió a su calculo en el procesador de datos SPSS V25, el cual arrojó una confiabilidad de 0,91 de coeficiente de Cronbach, considerándose confiable para su aplicación.

\section{Análisis de la información recopilada}

Se implementó la estadística descriptiva para el procesamiento de la información recopilada, diseñándose gráficos en donde se muestra el porcentaje de preferencia asumido por la muestra poblacional, empleándose el programa estadístico SPSS V25, para el cálculo de frecuencia y porcentajes, así como el programa Excel para la elaboración de los gráficos.

\section{RESULTADOS}

\section{Grafico 1}

Matemática inclusiva

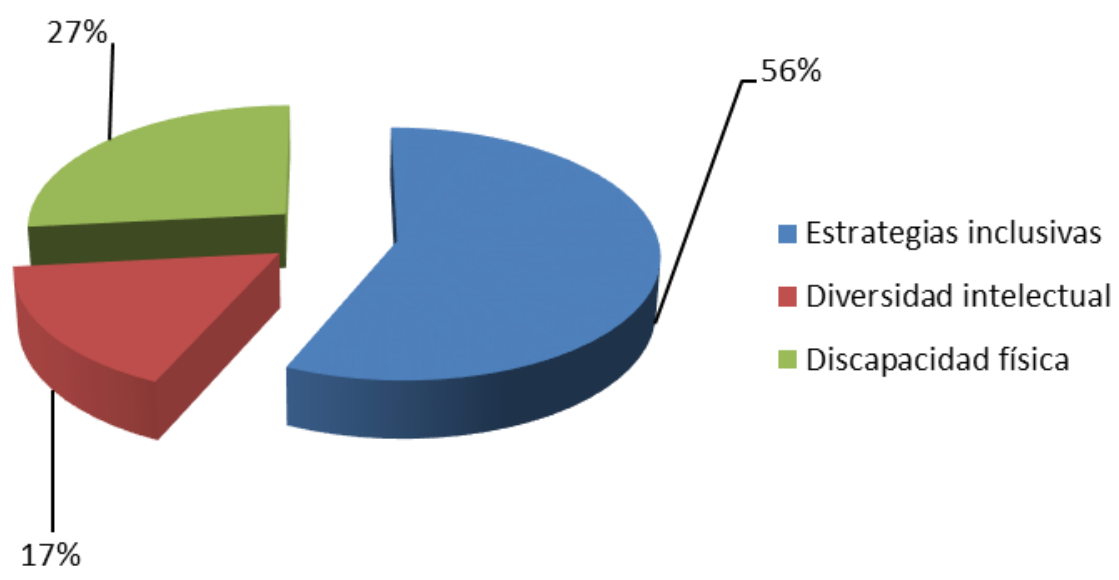


Ana Sofía Saltos-Cedeño; Patricio Alfredo Vallejo-Valdivieso; María Elena Moya-Martínez

El $56 \%$ considera que ha planificado en razón de incorporar estrategias inclusivas, el $17 \%$ propone estrategias para el abordaje de la diversidad intelectual y el $27 \%$ ha considerado estrategias para considerar los estudiantes que padecen discapacidad física para afrontar la modalidad virtual de aprendizaje.

\section{Grafico 2}

Actualización docente

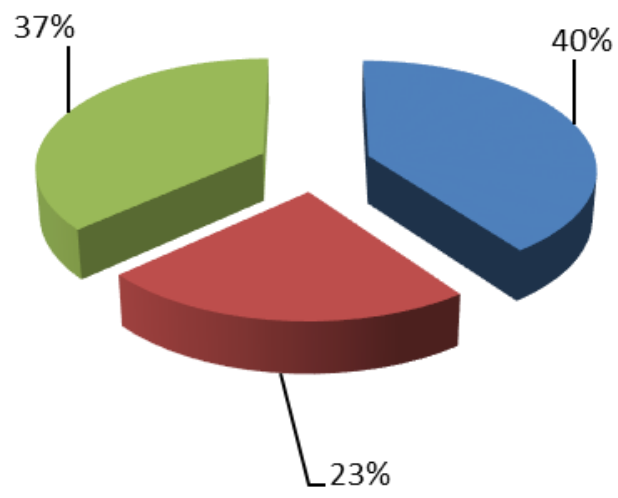

- Pariticipación en cursos durante la pandemia

- Formación previa a la pandemia

Autoformación

El $40 \%$ responde que ha participado en cursos de formación, programados durante la pandemia como parte de actualización para el nuevo período académico, el $23 \%$ considera que tiene adecuada formación para manejar la virtualidad antes de darse la emergencia por COVID-19 y el 23\% considera que su autorformación le ha permitido tener mayores conocimientos para afrontar los retos del nuevo ciclo educativo. 
Ana Sofía Saltos-Cedeño; Patricio Alfredo Vallejo-Valdivieso; María Elena Moya-Martínez

\section{Grafico 3}

Formación del estudiante

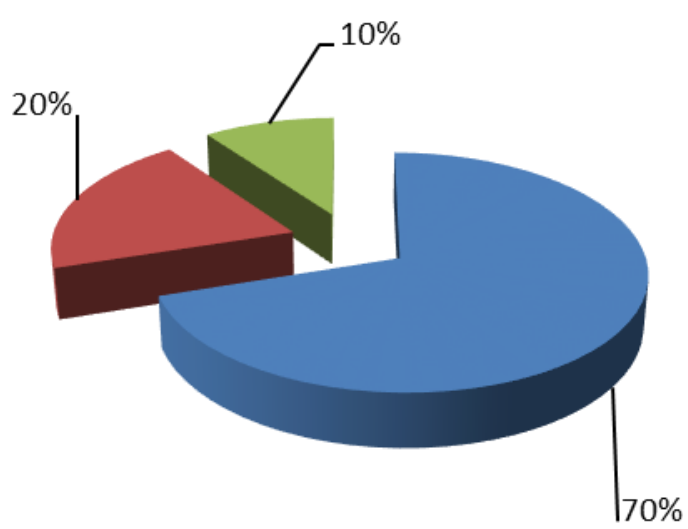

- Formar al estudiante sobre el uso de TIC

- Recursos didácticos TIC

Matemáticas en contexto

El 70\% considera pertinente formar a los estudiantes sobre el uso de las TIC con fines de promover un mejor aprendizaje, el $20 \%$ considera la actualización de recursos didácticos en TIC con fines de implementar un aprendizaje significativo y el $10 \%$ considera importante que el estudiante trabaje en su contexto social sobre el aprendizaje matemático.

\section{Grafico 4}

Innovación educativa

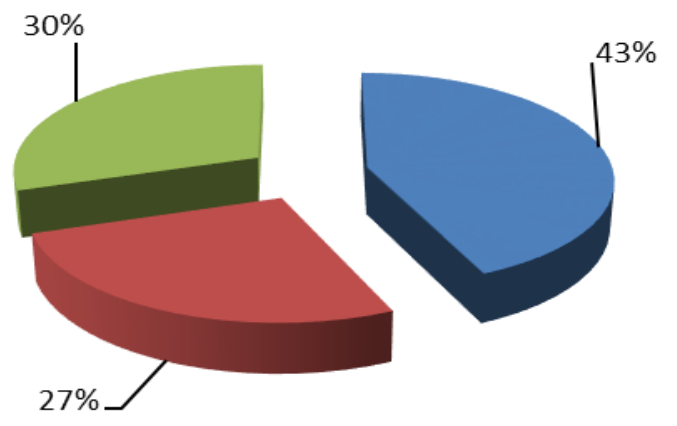

- Educación por competencias

- Educación por proyectos

- Educación basada en problemas 
Ana Sofía Saltos-Cedeño; Patricio Alfredo Vallejo-Valdivieso; María Elena Moya-Martínez

El $43 \%$ considera innovar en educación basada en competencias, el $27 \%$ plantea innovar mediante una educación basada en proyectos y el $30 \%$ considera el aprendizaje basado en problemas como métodos que permitan al estudiante formarse desde la virtualidad.

\section{DISCUSIÓN}

En cuanto al abordaje del área matemáticas inclusiva, el docente debe tener en consideración las discapacidades físicas e intelectuales que puedan tener los estudiantes con la finalidad de generar estrategias innovadoras e inclusivas que les permitan participar activamente (Aldana-Bermúdez, et al, 2018), siendo una oportunidad de inclusión durante el periodo virtual de educación por la pandemia, así mismo esta siuación permite promover una educación basada en la calidad del buen vivir (Rodríguez, Aguilar \& Apolo, 2018).

En complemento, (Cordero, 2015), destaca la implementación del método Braille para el aprendizaje de las matemáticas en estudiantes con diversidad funcional visual, posibilitando su integración al curso académico en equidad de condiciones, siendo indispensable que el docente conozca sobre el Braille, en su defecto la institución debe garantizar un asesor para que el estudiante no se vea mermado en su atención, destacando que hoy día se cuenta con este método a nivel tecnológico educacional. (Howard, et al, 2018), indica que en estudiantes con discapacidad intelectual, se debe diagnosticar individualmente para conocer las operaciones que pueden desarrollar.

Sin embargo, para que exista una educación inclusiva y de calidad, se debe fomentar la equidad como eje de integración, lo cual evitará el aislamiento de los estudiantes, así como tiende a mirar con perspectiva de respeto mutuo a los compañeros de clase (Briceño-de-Osorio, 2019), así mismo (Ayala-Asencio, et al, 2019), consideran necesario fomentar competencias interculturales en los estudiantes, lo cual permite tener mayor posibilidad de generar un clima de ciudadanía basada en tener presente las fortalezas para trabajar en equipo colaborativamente. 


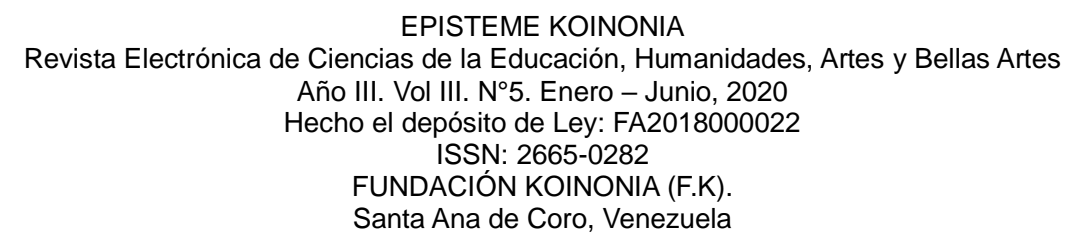

Ana Sofía Saltos-Cedeño; Patricio Alfredo Vallejo-Valdivieso; María Elena Moya-Martínez

La actualización docente es fundamental para estar a la altura de los retos, desafíos a enfrentar en la enseñanza de la matemática, desde una perspectiva innovadora de la educación (Díaz-Pinzón, 2018), confronta la enseñanza tradicional y evidencia la necesidad de incorporar estrategias como la simulación, situación que podría favorecer el aprendizaje en los estudiantes para lo cual se requiere que el docente tenga experiencia en el manejo de programas que posibiliten esta tendencia educativa (Cistac, Bongianino, Filippi \& Kovac, 2009).

En este sentido, se tiene en cuenta la propuesta de (Romero \& Villasmil, 2017), sobre la necesidad de repensar la formación epistemológica de los futuros docentes de educación, siendo necesaria involucrar a los profesionales en ejercicio profesional con la finalidad de adecuarse al enfoque de constructivismo social mediado por las tecnologías educativas (Vallejo-Valdivieso, et al, 2019), valoran el conectivismo como una filosofía necesaria para comprender la conectividad global del mundo actual, donde lo educativo forma parte del mismo, siendo pertinente que los educadores tengan en consideración la perspectiva de red para la planificación y evalaución de los aprendizajes, siendo la HUMANHOLOPRAXIS, una corriente que permite integrar un proceso reflexivo de formación permenente en el docente (Adames-Bueno \& Rodríguez, 2018).

La formación del estudiante es esencial para contribuir a la generación de un aprendizaje en concordancia con las metas planteadas por las políticas educativas (Zempoalteca-Durán, et al, 2017), promoviéndose la posibilidad de evitar la deserción escolar o el bajo rendimiento académico (López-Mero, et al, 2015), considera que el apoyo familiar juega un rol fundamental para apoyar al estudiante en lograr las metas académicas.

Así mismo, (Eljuri-Blanco \& Villasmil-Yánez, 2018), consideran importante la generación de una cultura organizacional que fomente en la institución la formación permanente tanto de docentes como estudiantes en competencias extracurriculares, siendo hoy día necesario propiciar en los estudiantes, el uso de las TIC con fines educativos, por 
cuanto esto permite promover un aprendizaje significativo en función de cumplir con los lineamientos planteados en el período por confinamiento por COVID-19, aunado a la necesidad de estimular las estructuras mentales para el manejo asertivo de las emociones por parte de los estudiantes, por cuanto esto contribuye a una mejor integración escolar en función de los retos académicos actuales (Salvatierra-Tumbaco, Vallejo-Valdivieso \& Moya-Martínez, 2019).

En el área innovación educativa, se tiene que el aprendizaje basado en problemas, por competencias y por proyectos, tienen como fundamento la centralidad en el estudiante (Peche-Cruz \& Giraldo-Supo, 2019), siendo que este enfoque promueve como principal innovación, la investigación, por lo tanto, tanto docentes como alumnos se constituyen en un equipo de trabajo con la finalidad de conciliar en conjunto el aprendizaje, bajo la premisa de la constante indagación reflexiva en la búsqueda de información, situación que tiene como soporte el uso de las TIC, sin embargo es necesario vigilar la aplicación de estas metodologías, por cuanto pueden ser mal empleadas y distorsionarse la esencia de las mismas en detrimento de la calidad de la educación (Gutiérrez, Rodríguez \& Isea Argüelles, 2019).

En complemento, se destaca la importancia del rol que juegan los directivos de las instituciones en el apoyo de promover una educación de calidad en consonancia con los nuevos tiempos afectados por el confinamiento por COVID-19, por cuanto ellos como líderes educativos están llamados a la concertación de fortalezas para promover la transcendencia a una sociedad basada en valores construidos desde el aprendizaje promovido por el sistema educativo (Lugo \& Villasmil Ferrer, 2019). Por otro lado, (Montiel-Espinosa, 2016), indica la necesidad de innovar educativamente para el fomento de un aprendizaje matemático en conformidad a los nuevos escenarios sociales en donde se ve envuelto el estudiante, siendo los cursos de posgrados, un espacio para propiciar tales transformaciones en los profesionales de la educación. 
Ana Sofía Saltos-Cedeño; Patricio Alfredo Vallejo-Valdivieso; María Elena Moya-Martínez

\section{CONCLUSIONES}

La innovación en educación matemática de básica superior durante confinamiento por COVID-19 en el Ecuador, debe centrarse en un aprendizaje mediado por TIC mediante metodologías centradas en el estudiante como aprendizaje basado en problemas, con lo cual, el docente tiene la posibilidad de diseñar estrategias en conformidad a las necesidades del entorno social donde habita el estudiante, para lo cual, puede desarrollar proyectos en donde se involucren las operaciones matemáticas en consonancia con los recursos disponibles para tal fin, con lo cual, además se promueven una serie de competencias como la conciencia ecológica, comunicación intercultural, reflexión, comprensión, sumado a la indagación investigativa como medio de búsqueda de información que contribuya a la resolución del problema planteado para el desarrollo del proyecto. Así mismo, se plantean que:

Los docentes deben actualizarse en competencias virtuales que le permitan procesar el aprendizaje de modo efectivo en los estudiantes.

Se debe capacitar a los estudiantes en las TIC para que puedan empoderarse de las estrategias y recursos didácticos basados en la virtualidad.

El apoyo familiar es fundamental para el acompañamiento y motivación de los estudiantes con fines de adaptarse a la educación virtual.

Las diversidades y discapacidades de los estudiantes deben ser tenidas en cuenta para el diseño de las estrategias de aprendizaje con la finalidad de generar una educación inclusiva y de calidad para el buen vivir.

\section{FINANCIAMIENTO}

No monetario 
Ana Sofía Saltos-Cedeño; Patricio Alfredo Vallejo-Valdivieso; María Elena Moya-Martínez

\section{AGRADECIMIENTOS}

A los Directivos y docentes de la Unidad Educativa Fiscomisional San Francisco de Sales, ubicada en la provincia de Manabí-Ecuador, por el apoyo en el desarrollo de la investigación.

\section{REFERENCIAS}

Aldana-Bermúdez, E, Gutiérrez-Zuluaga, H, \& Wagner-Osorio, G. (2018). Formación de profesores para una educación matemática en y para la diversidad. [Teacher training for a mathematical education in and for diversity]. Sophia, 14(1), 6574. https://dx.doi.org/10.18634/sophiaj.14v.1i.823

Ausín, V, Abella, V, Delgado, V, \& Hortigüela, D. (2016) Aprendizaje basado en proyectos a través de las TIC: una experiencia de innovación docente desde las aulas universitarias. [Project-Based Learning through ICT. An Experience of Teaching Innovation from University Classrooms]. Formación universitaria , 9 (3), 31-38. https://dx.doi.org/10.4067/S0718-50062016000300005

Adames-Bueno, S., \& Rodríguez, C. (2018). HUMANHOLOPRAXIS: Hallazgo de la práctica pedagógica del docente de matemática en la Universidad Politécnica Territorial Alonso Gamero. [HUMANHOLOPRAXIS: Finding of the pedagogical practice of the mathematics teacher at the Alonso Gamero Polytechnic Territorial University]. Revista Arbitrada Interdisciplinaria Koinonía, 3(6), 38-61. Recuperado de https://n9.cl/12bc

Ayala-Asencio, C, Garro-Aburto, L, Sanabria-Boudri, F, Aldana-Zavala, J, Colina-Ysea, F, \& Albites-Sanabria, J. (2019). Competencias interculturales en el proceso de capacitación en investigación en una universidad privada de Lima, Perú. [ntercultural competences in the research training process at a private university in Lima, Peru]. Revista Espacios, Vol. 40 (№ 44) Año 2019. P. 13. Recuperado de: https://cutt.ly/atPiXON

Briceño-de-Osorio, A. (2019). Aprendizaje de lenguas para la diversidad cultural. [Language learning for cultural diversity]. EPISTEME KOINONIA, 2(4), 53-71. http://dx.doi.org/10.35381/e.k.v2i4.524

Calvo, G. (2015). La formación de docentes para la inclusión educativa. [Teacher training for inclusive education]. Páginas De Educación, 6(1), 19-35. https://n9.cl/umb0 
Calvo-Cereijo, M. (2019). Pensamiento complejo y transdisciplina. [Complex thinking and transdiscipline]. Sophia, Colección de Filosofía de la Educación, (26), 307326. https://dx.doi.org/10.17163/soph.n26.2019.09

Carranza-Alcántar, M. del R. (2018). Enseñanza y aprendizaje significativo en una modalidad mixta: percepciones de docentes y estudiantes. [Significant teaching and learning in a blended learning: perceptions of teachers and students]. RIDE Revista Iberoamericana Para La Investigación y El Desarrollo Educativo, 8(15), 898 - 922. https://doi.org/10.23913/ride.v8i15.326

Cartes-Velasquez, R, \& Moraga, J. (2016). Pautas de chequeo, parte III: STROBE y ARRIVE. [Cheklists, part III: STROBE and ARRIVE]. Revista chilena de cirugía, 68(5), 394-399. https://dx.doi.org/10.1016/j.rchic.2015.12.003

Cistac, G, Bongianino, R, Filippi, J, \& Kovac, F. (2009). La Simulación como Medio de Interrelación entre Herramientas Matemáticas y Procesos Tecnológicos. [Simulation as Interrelationship between Mathematic Tools and Technological Process]. Formación universitaria, 2(5), 312. https://dx.doi.org/10.4067/S0718-50062009000500002

Cordero, D. (2015). La matemática mediante el método braille en niños (as) con diversidad funcional visual. [Mathematics using the braille method in children with visual functional diversity]. CIENCIAMATRIA, 1(1), 73-88. https://doi.org/10.35381/cm.v111.63

Creamer, M. (2020). Plan Educativo Covid-19 se presentó el 16 de marzo. [Covid-19 Educational Plan was presented on March 16]. Recuperado de https://url2.cl/fu54M

De-La-Peña-Álvarez, C., \& Bernabéu-Brotóns, E. (2018). Dislexia y discalculia: una revisión sistemática actual desde la neurogenética. [Dyslexia and dyscalculia: a current systematic review from neurogenetics]. Universitas Psychologica, 17(3), 1-11. https://doi.org/10.11144/Javeriana.upsy17-3.ddrs

Díaz-Pinzón, J. E. (2018). Aprendizaje de las Matemáticas con el uso de Simulación. [Learning Mathematics with the use of Simulation]. Sophia, 14(1), 2230. https://doi.org/10.18634/sophiaj.14v.1i.519

Eljuri-Blanco, A., \& Villasmil-Yánez, M. (2018). Cultura organizacional en docentes de educación básica. [Organizational culture in basic education teachers]. EPISTEME KOINONIA, 1(2), 4-17. http://dx.doi.org/10.35381/e.k.v1i2.508 
Estrada, M, Monferrer, D, \& Moliner, M. (2016). El Aprendizaje Cooperativo y las Habilidades Socio-Emocionales: Una Experiencia Docente en la Asignatura Técnicas de Ventas. [Cooperative Learning and Socio-Emotional Skills: A Teaching Experience in Sales Techniques Course]. Formación universitaria, 9(6), 43-62. https://dx.doi.org/10.4067/S0718-50062016000600005

García-Navarro, D. X., Massani-Enríquez, D, \& Bermúdez-López, M. I. L. (2016). La educación inclusiva en la formación de profesionales de la educación. [Inclusive education in the training of education professionals]. Revista Conrado, 12(52). 610. Recuperado de https://url2.cl/iGFvm

Gutiérrez, N., Rodríguez., N., \& Isea Argüelles, J. (2019). La formación por competencia ¿un enfoque mal implementado en las instituciones educativas universitarias? [Competency-based training: a poorly implemented approach in university educational institutions?]. $\quad$ CIENCIAMATRIA, 5(8), 21-49. https://doi.org/10.35381/cm.v5i8.86

Grisales-Aguirre, A. (2018). Uso de recursos TIC en la enseñanza de las matemáticas: retos y perspectivas. [Use of ICT resources in the teaching of mathematics: challenges and perspectives]. Entramado, 14(2), 198-214. https://doi.org/10.18041/1900-3803/entramado.2.4751

Hernández, R., Fernández, C., \& Baptista, M. (2014). Metodología de la Investigación. [Research Methodology]. Mexico DF: McGraw-Hill / Interamericana Editores, S.A. DE C.V.

Herrera, J, Parrilla, Á, Blanco, A, \& Guevara, G. (2018). La Formación de Docentes para la Educación Inclusiva. Un Reto desde la Universidad Nacional de Educación en Ecuador. [Teacher Training for Inclusive Education. A Challenge from the National University of Education in Ecuador]. Revista latinoamericana de educación inclusiva, 12(1), 21-38. https://dx.doi.org/10.4067/S0718$\underline{73782018000100021}$

Howard, S., San-Martin, C., Salas, N., Blanco, P., \& Díaz, C. (2018). Oportunidades de aprendizaje en matemáticas para estudiantes con discapacidad intelectual. [Math Learning Opportunities for Students with Learning Disabilities]. Revista Colombiana de Educación, (74), 197-219. https://doi.org/10.17227/rce.num74$\underline{6906}$

Instituto Nacional de Evaluación Educativa (2018). La educación en Ecuador: Logros alcanzados y nuevos desafíos. [Education in Ecuador: Achievements and new challenges]. Recuperado de https://url2.cl/gQg54 
Ana Sofía Saltos-Cedeño; Patricio Alfredo Vallejo-Valdivieso; María Elena Moya-Martínez

Islas-Torres, C. (2018). La implicación de las TIC en la educación: Alcances, Limitaciones y Prospectiva. [The role of ICT in education: Applications, Limitations, and Future Trends]. RIDE Revista Iberoamericana Para La Investigación Y El Desarrollo Educativo, 8(15), $861 \quad$ - 876. https://doi.org/10.23913/ride.v8i15.324

Jiménez-Galán, Y. I. (2017). Innovación educativa y docencia ¿falla el protagonista?: el caso ESCOM. [Educational innovation ¿Does the protagonist fail?: the ESCOM case]. RIDE Revista Iberoamericana Para La Investigación Y El Desarrollo Educativo, 8(15), 710 - 734. https://doi.org/10.23913/ride.v8i15.317

López-Mero, P., Barreto-Pico, A., Mendoza-Rodríguez, E., \& Del-Salto-Bello, M. (2015). Bajo rendimiento académico en estudiantes y disfuncionalidad familiar. [Low academic performance in students and family dysfunction]. MEDISAN, 19(9). 1163-1166. Recuperado de https://url2.cl/mWYG1

Lugo, N., \& Villasmil Ferrer, J. (2019). Liderazgo directivo como factor de mejoramiento en la calidad educativa. [Managerial leadership as a factor in improving

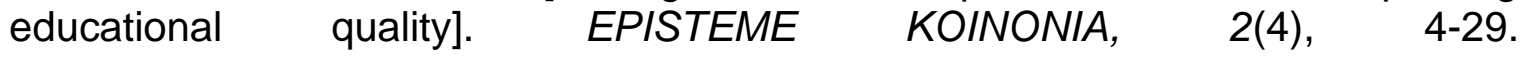
http://dx.doi.org/10.35381/e.k.v2i4.521

Mendoza, H., Burbano, V, \& Valdivieso, M. (2019). El Rol del Docente de Matemáticas en Educación Virtual Universitaria. Un Estudio en la Universidad Pedagógica y Tecnológica de Colombia. [The Role of the Teacher of Mathematics in Virtual University Education. A Study in the Pedagogic and Technologic University of Colombia]. Formación universitaria, 12(5), 5160. https://dx.doi.org/10.4067/S0718-50062019000500051

Ministerio de Educación del Ecuador (2016). Currículo de los niveles de educación obligatoria. [Curriculum of the levels of compulsory education]. Recuperado de https://url2.cl/Gn8Jr

Montiel-Espinosa, G. (2016). Condiciones para la innovación educativa en el posgrado. El caso de la Maestría en Enseñanza de las Matemáticas en la Educación Secundaria en Oaxaca. [Conditions for educational innovation at the postgraduate level. The case of the Master's Degree in Mathematics Teaching in Secondary Education in Oaxaca]. Perfiles educativos, 38 (spe), 101115. Recuperado de https://n9.cl/tgywh

Muñoz-Camacho, S, Gallardo, T, Muñoz-Bravo, M, \& Muñoz-Bravo, C. (2018). Probabilidad de Deserción Estudiantil en Cursos de Matemáticas Básicas en 


\section{Ana Sofía Saltos-Cedeño; Patricio Alfredo Vallejo-Valdivieso; María Elena Moya-Martínez}

Programas Profesionales de la Universidad de Los Andes-Venezuela. [Probability of student abandonment in basic mathematics courses in professional programs at the University of Los Andes-Venezuela]. Formación universitaria, 11(4), 3342. https://dx.doi.org/10.4067/S0718-50062018000400033

Orrantia, J. (2006). Dificultades en el aprendizaje de las matemáticas: una perspectiva evolutiva. [Difficulties in learning mathematics: an evolutionary perspective]. Revista Psicopedagogia, 23(71), 158-180. Recuperado de https://url2.cl/JplR8

Pascua-Cantarero, P. (2016). Factores relacionados con la deserción en el primer y segundo año de estudio en la carrera de Enseñanza de la Matemática de la Universidad Nacional de Costa Rica. [Factors Related to Dropping Out in the Freshman and Sophomore Years in the Career of Teaching Mathematics at Universidad Nacional de Costa Rica]. Revista Electrónica Educare, 20(1), 96118. https://dx.doi.org/10.15359/ree.20-1.5

Peche-Cruz, H., \& Giraldo-Supo, V. (2019). El Aprendizaje Flip Learning centrado en el estudiante como generador de calidad educativa. [Student-centered Flip Learning as a generator of educational quality]. Revista Arbitrada Interdisciplinaria Koinonía, 4(8), 427-450. http://dx.doi.org/10.35381/r.k.v4i8.293

Ponce-Díaz, R. (2017). ¿Cómo innovar en la enseñanza de las matemáticas? [How to innovate in the teaching of mathematics?]. Recuperado de https://url2.cl/6fE2l

Rodríguez-Pautt, B. (2014). Entornos virtuales como estrategia para la enseñanza y el aprendizaje de las matemáticas. [Virtual environments as a strategy for teaching and learning mathematics]. Recuperado de https://url2.cl/bvvi5

Rodríguez, M, Aguilar, J, \& Apolo, D. (2018). El Buen vivir como desafío en la formación de maestros: aproximaciones desde la Universidad Nacional de Educación del Ecuador. [Good living as a challenge in teacher training: approaches from the National University of Education of Ecuador]. Revista mexicana de investigación educativa, 23(77), 577-596. Recuperado de https://url2.cl/R5spP

Romero, M., \& Villasmil, J. (2017). Repensar la formación docente. Hacia el encuentro de una nueva perspectiva epistémica para su abordaje y resignificación. [Rethink teacher training. towards the meeting of a new epistemic perspective for its approach and resignification]. CIENCIAMATRIA, 3(5), 133-149. https://doi.org/10.35381/cm.v3i5.17

Said-Hung, E, Valencia-Cobos, J, \& González-Prieto, E. (2017). La promoción de experiencias de innovación educativa en TIC en los centros escolares: Caso 
región Caribe colombiana. [The promotion of educational innovation experiences in ICT in schools: Case of the Colombian Caribbean region]. Estudios pedagógicos (Valdivia), 43(1), 457-473. https://dx.doi.org/10.4067/S0718$\underline{07052017000100026}$

Salvatierra-Tumbaco, D., Vallejo-Valdivieso, P., \& Moya-Martínez, M. (2019). Estructuras mentales y competencias emocionales en estudiantes universitarios. [Mental structures and emotional competencies in university students]. CIENCIAMATRIA, 5(1), 118-132. https://doi.org/10.35381/cm.v5i1.260

Travieso-Valdés, D. \& Ortíz-Cardenas, T. (2018). Aprendizaje basado en problemas y enseñanza por proyectos: Alternativas diferentes para enseñar. Revista Cubana de Educación Superior, 37(1), 124-133.

Vallejo-Valdivieso, P., Zambrano-Pincay, G., Vallejo-Pilligua, P., \& Bravo-Cedeño, G. (2019). Importancia del Conectivismo en la inclusión para mejorar la Calidad Educativa ante la tecnología moderna. [mportance of Connectivism in inclusion to improve Educational Quality in the face of modern technology]. Revista Arbitrada Interdisciplinaria Koinonía, 4(8), 523-543. http://dx.doi.org/10.35381/r.k.v4i8.297

Vega-Vega, J, Niño-Duarte, F, \& Cárdenas, Y. (2015). Enseñanza de las matemáticas básicas en un entorno e-Learning: un estudio de caso de la Universidad Manuela Beltrán Virtual. [Teaching basic mathematics in an e-Learning environment: a case study from the Manuela Beltrán Virtual University]. Revista EAN, (79), 172187. Recuperado de https://url2.cl/ruDmK

Zempoalteca-Durán, B, Barragán-López, J, González-Martínez, J, \& Guzmán-Flores, T. (2017). Formación en TIC y competencia digital en la docencia en instituciones públicas de educación superior. [ICT training and digital competence in teaching in public institutions of higher education]. Apertura (Guadalajara, Jal.), 9(1), 8096. https://dx.doi.org/10.32870/ap.v9n1.922 